\title{
Cardiorespiratory Fitness Impact on All-Cause Mortality in Prediabetic Veterans
}

\author{
Eric S. Nylen ${ }^{\mathrm{a}, \mathrm{c}}$, David Ni ${ }^{\mathrm{a}}$, Jonathan Myers ${ }^{\mathrm{b}}$, Manchin Chang ${ }^{\mathrm{a}}$, Mary T. Plunkett ${ }^{\mathrm{a}}$, \\ Peter Kokkinos ${ }^{\mathrm{b}}$
}

\begin{abstract}
Background: The objective of this study was to evaluate the role of cardiorespiratory fitness (CRF) on all-cause mortality in prediabetic veterans.

Methods: In this prospective cohort study, CRF was calculated from metabolic equivalents (METs) obtained from routine exercise tolerance testing in a cohort of 1,118 prediabetic veterans. Four fitness categories were established: low-fit $(<5.8 \mathrm{METs}$; lower 25 th percentile), mild-fit (5.8 - 7 METs), moderate-fit (7.1 - 8.5 METs) and high-fit ( $>8.5$ METs; $>75$ th percentile). Date of death was verified from the Veterans Affairs Beneficiary Identification and Record Locator System File.
\end{abstract}

Results: The mean follow-up period was 7.7 years $(8,610$ personyears) and there were a total of 251 deaths, averaging 29.1 events per 1,000 person-years. An inverse and graded association between $\mathrm{CRF}$ and mortality risk was observed $(\mathrm{P}=0.002)$. For every 1-MET increase in $\mathrm{CRF}$, the adjusted mortality was lowered by $13 \%$ (hazard ratios $(\mathrm{HR})=0.87$; CI: $0.81-0.94, \mathrm{P}<0.001)$. The mild-, moderateand high-Fit subjects had mortality reductions of $32 \%(\mathrm{HR}=0.68 ; \mathrm{CI}$ : $0.50-0.94, \mathrm{P}=0.02), 40 \%(\mathrm{HR}=0.60 ; \mathrm{CI}: 0.41-0.87, \mathrm{P}=0.007)$ and $54 \%(\mathrm{HR}=0.46$; CI: $0.30-0.70, \mathrm{P}<0.001)$ respectively.

Conclusions: A strong inverse and graded association between CRF and mortality risk was observed in these prediabetic veterans. Mortality risk was $13 \%$ lower for every 1 -MET increase in CRF and approximately $50 \%$ lower in high-fit individuals (exercise capacity $>8.5$ METs) compared to least-fit individuals. With increasing incidence of prediabetes as well as diminished response to preventative lifestyle modifications, enhanced CRF should be advocated in prediabetic individuals.

Keywords: Cardiorespiratory fitness; Prediabetes; All-cause mortality

Manuscript accepted for publication June 04, 2015

${ }^{a}$ VAMC, Washington, DC, USA

bVAMC, Palo Alto, CA, USA

${ }^{\mathrm{c} C o r r e s p o n d i n g ~ A u t h o r: ~ E r i c ~ N y l e n, ~} 50$ Irving St. NW, Rm. GE246, VAMC, Washington, DC 20422, USA. Email: enylen@gmail.com

doi: http://dx.doi.org/10.14740/jem284w

\section{Introduction}

The CDC estimates that 79 million Americans (approximately one in three) have prediabetes, a condition associated with developing type 2 diabetes (DM2) which is a major cause of cardiovascular disease (CVD) and strokes, and is the seventh leading cause of death in the United States [1,2]. The US Diabetes Prevention Program, which compared active lifestyle intervention or metformin to standard lifestyle in overweight Americans with impaired glucose tolerance (IGT), demonstrated that active lifestyle reduced the incidence of DM2 by $58 \%$ [3]. Follow-up of this cohort showed that diabetes risk was $56 \%$ lower in patients who returned to normal glucose tolerance [4]. A number of different studies have given support to the notion that physical exercise and weight loss significantly impact prevention or delay of DM2 in patients with IGT [510]. Though lifestyle intervention has become a routine recommendation for patients with prediabetes, there is a paucity of data regarding mortality outcome [8]. Recently, the Da Qing study showed a significant decrease in cardiovascular and allcause mortality [11] and cardiorespiratory fitness (CRF) was a strong modulator of survival in a large relatively healthy prediabetic cohort [12].

The Veterans Health Administration utilizes an electronic health information database of patients given equal access to high quality health care independent of financial status. The objective of this study was to evaluate the role of CRF on allcause mortality in veterans with prediabetes.

\section{Materials and Methods}

Between January 1995 and December 2010, a total of 1,118 prediabetic veterans underwent symptom-limited exercise tolerance testing (predominantly Bruce and Modified Bruce Protocols) in Washington, DC, and Palo Alto, California, for routine testing or to assess for exercise-induced ischemia as previously described in detail $[13,14]$. All demographic, clinical information and vital signs were obtained from the patient's records within 3 months of the exercise tolerance test. BMI was calculated as weight in kilograms divided by the square of height in meters. Demographic data are included in Table 1. Based on their calculated peak metabolic equivalents (METs), four CRF categories were established as follows: least-fit $(<$ 
Table 1. Population Demographics

\begin{tabular}{lllll}
\hline & Least-fit (< 5.8 METs) & Low-fit (5.8 - 7 METS) & Moderate-fit (7.1 - 8.4 METs) & High-fit ( $\geq$ 8.5 METs) \\
\hline Number of patients & 289 & 292 & 265 & 272 \\
Events & $101(34.9 \%)$ & $66(22.6 \%)$ & $48(18.1 \%)$ & $36(13.2 \%)$ \\
Age & $65.7 \pm 10.2$ & $60.3 \pm 10.2$ & $56.7 \pm 9.5$ & $52.4 \pm 8.5$ \\
BMI & $30.4 \pm 6.6$ & $30.6 \pm 5.5$ & $30.2 \pm 5.1$ & $28.4 \pm 4.1$ \\
Gender & & & 263 \\
$\quad$ Male & 272 & 276 & 10 & 9 \\
$\quad$ Female & 17 & 16 & & 72 \\
Race & & & 76 & 199 \\
$\quad$ White & 75 & 67 & 189 & $157(57.9 \%)$ \\
$\quad$ Black or other & 214 & 225 & $176(66.4 \%)$ & $37(19.7 \%)$ \\
Hypertension & $223(77.2 \%)$ & $214(73.3 \%)$ & $39(21.7 \%)$ & $108(40.6 \%)$ \\
Smoking & $47(24.0 \%)$ & $55(25.9 \%)$ & $117(45.0 \%)$ & $196.0 \pm 37.9$ \\
Dyslipidemia & $109(38.8 \%)$ & $124(42.8 \%)$ & $195.6 \pm 49.5$ & $135.4 \pm 98.0$ \\
Total cholesterol (mg/dL) & $187.7 \pm 43.0$ & $193.7 \pm 40.8$ & $167.3 \pm 198.5$ & $122.1 \pm 34.0$ \\
Triglycerides (mg/dL) & $162.0 \pm 175.3$ & $148.0 \pm 110.1$ & $120.5 \pm 40.1$ & $50.3 \pm 14.6$ \\
LDL-C (mg/dL) & $113.7 \pm 34.3$ & $121.2 \pm 47.1$ & $47.8 \pm 13.9$ & \\
HDL-C (mg/dL) & $47.3 \pm 13.3$ & $48.5 \pm 14.2$ & & \\
\hline
\end{tabular}

Age, BMI, total cholesterol, triglycerides, LDL-C and HDL-C are expressed as means \pm SD. Hypertension defined as a systolic blood pressure $>130 \mathrm{~mm} \mathrm{Hg}$ or a diastolic blood pressure $>80$. Dyslipidemia defined as a total cholesterol $>200 \mathrm{mg} / \mathrm{dL}$, LDL > $130 \mathrm{mg} / \mathrm{dL}$ or triglyceride > $199 \mathrm{mg} / \mathrm{dL}$.

5.8 METs; lowest 25th percentile), low-fit (5.8 - 7 METs), moderate-fit (7.1 - 8.4 METs) and high-fit ( $\geq 8.5 \mathrm{METs} ;>75$ th percentile).

The date of death was verified from the Veterans Affairs Beneficiary Identification and Record Locator System File. Cox proportional hazard analyses were applied to assess the risk among the fitness categories. The analyses were adjusted for age, BMI, history of CVD, cardiovascular medications (ACE inhibitors, beta blockers, calcium channel blockers, diuretics and statins) as well as risk factors (hypertension, dyslipidemia and smoking).

The least-fit category was used as the reference group. All hypotheses were two-sided and P-values $<0.05$ were considered statistically significant. All statistical analyses were performed using SPSS software (Version 15.0; SPSS, Chicago,
IL).

\section{Results}

In a mean follow-up period of 7.7 years $(8,610$ person-years), there were a total of 251 deaths, averaging 29.1 events per 1,000 person-years. The composition of the quartiles was similar in terms of gender, race, incidence of dyslipidemia, as well as cholesterol values (Table 1). Higher CRF category patients were noted to have a mean age which was generally younger than the least-fit patients, although there did appear to be an overlap regarding age stratification. It was noted that for every 1 year difference, there was a $1.9 \%$ increased risk in mortality or a 10 -year mortality risk of $21 \%$. Additionally, the presence

Table 2. Variables in the Equation

\begin{tabular}{llll} 
& HR & CI & P-value \\
\hline Age & 1.019 & $1.005-1.032$ & 0.006 \\
CVD & 2.018 & $1.519-2.681$ & $<0.001$ \\
Lowest-fit (< 5.8 METs) & 1.000 & Reference & Reference \\
Low-fit (5.8 - 7 METs) & 0.683 & $0.496-0.941$ & 0.020 \\
Moderate-fit (7.1 - 8.4 METs) & 0.599 & $0.413-0.867$ & 0.007 \\
High-fit (> 8.5 METs) & 0.463 & $0.304-0.705$ & $<0.001$ \\
\hline
\end{tabular}

Using lowest-fit as a reference, adjusted HRs for mortality were determined for contributions by age and presence of CVD. 
Table 3. Variables Not in the Equation

\begin{tabular}{ll}
\hline & P-value \\
\hline BMI & 0.360 \\
Cardiovascular medications & 0.468 \\
Smoking status & 0.154 \\
Hypertension & 0.774 \\
\hline
\end{tabular}

Cardiovascular Medications refers to the presence of one of the following classes of medications at the time of ETT-beta blocker, calcium channel blocker, ACE inhibitor, angiotensin receptor blocker, diuretic, aspirin or statin.

of CVD (ranging from a history of NSTEMI to four vessel bypass) essentially doubled the risk of mortality (Table 2). As such, age and CVD are adjusted for in the final analysis. BMI, smoking status, usage of cardiovascular medications and presence of hypertension on the other hand were not statistically significant in correlation with overall mortality (Table 3 ).

An inverse and graded association between CRF and mortality risk was observed ( $\mathrm{P}=0.002$ for trend). For every 1 -MET increase in $\mathrm{CRF}$, the adjusted all-cause mortality was lowered by $13 \%$ (hazard ratios $(\mathrm{HR})=0.87$; CI: $0.81-0.94, \mathrm{P}<0.001)$. Compared to the least-fit individuals, all-cause mortality risk was reduced by $32 \%$ in low-fit individuals $(\mathrm{HR}=0.68$; CI: $0.50-0.94, \mathrm{P}=0.002$ ), by $40 \%$ in moderate-fit individuals $(\mathrm{HR}=0.60 ; \mathrm{CI}: 0.41-0.87, \mathrm{P}=0.007)$ and by $54 \%$ in high-fit individuals ( $\mathrm{HR}=0.46$; $\mathrm{CI}: 0.30-0.70, \mathrm{P}<0.001)$. A summary of these results is illustrated in Figure 1.

\section{Discussion}

We observed a strong inverse and graded association between CRF and mortality risk with a $13 \%$ decrease for every 1 -MET increase in fitness. All-cause mortality risk was markedly reduced by $32 \%$ when comparing the low-fit and least-fit individuals and by approximately $50 \%$ when comparing high-fit and least-fit individuals. Notably, BMI and CVD factors such as cardiovascular medications, smoking status and hypertension did not appear to be significant factors in the correlation between CRF and mortality risk. These results conform to our prior studies in several chronic illnesses [14-16].

The American Diabetes Association currently recommends that DM2 persons undertake at least 150 min per week of moderate-to-vigorous aerobic exercise spread out at least 3 days during the week with no more than 2 consecutive days between bouts of physical activity, and that persons with DM2 should undertake moderate-to-vigorous resistance training at least 2 - 3 days per week [17]. More recently, the Australian Exercise and Sport Science promotes 210 min of moderate-intensity exercise or $125 \mathrm{~min}$ of more intense exercise per week that should also include resistance training [18]. Using these exercise parameters has been shown to be efficacious in several diabetes prevention programs. The Diabetes Prevention Program (DPP) included 3,819 prediabetics who were randomly

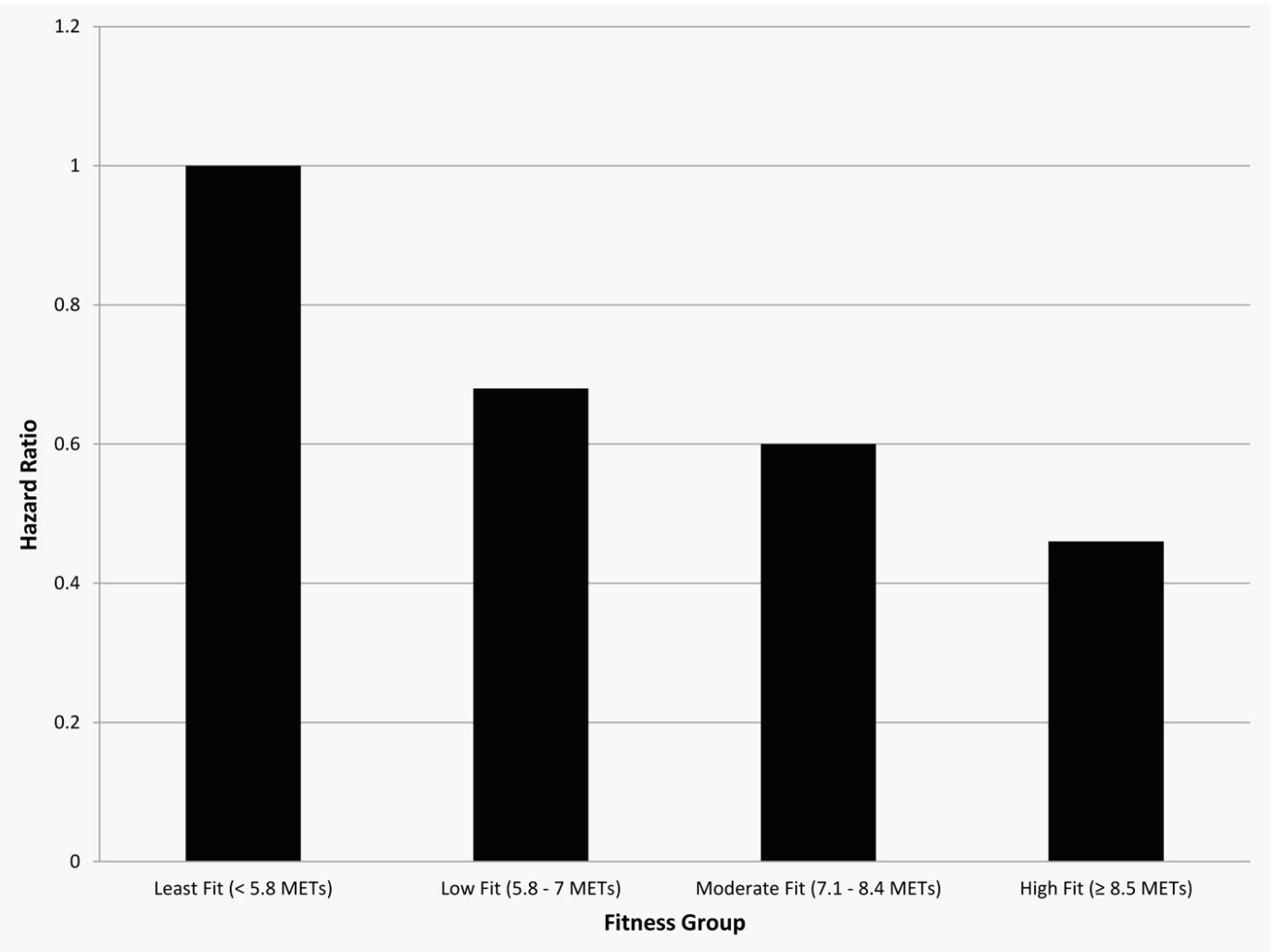

Figure 1. Relative risk for all-cause mortality according to fitness group. 
assigned to an intensive lifestyle intervention group comprising of $7 \%$ weight loss and at least 150 min of physical activity per week, metformin with standard lifestyle advice and a control group. A 58\% reduction in the incidence of DM2 was seen in the intensive lifestyle intervention group compared with the control group, and it was further noted that exercise was also more effective than metformin in preventing progression to diabetes (NNT 6.9 vs. 13.9) [3,4]. In the Swedish 6-year Malmo feasibility study, 415 men of ages 47 - 49 years participated in a large population-based screening program. Persons with diabetes, prediabetes or normal glucose tolerance were non-randomly assigned to lifestyle intervention or usual care groups. After 6 years, the $10.6 \%$ of the prediabetic patients in the lifestyle intervention group developed diabetes compared to $28.6 \%$ in the usual care group [5]. In the Chinese Da Qing Impaired Glucose Tolerance and Diabetes Study, 557 prediabetic patients were randomly assigned to a control group or one of three intervention groups involving diet, exercise, or diet and exercise. After 6 years, the incidence of diabetes in the control group was $67.7 \%$ compared with $43.8 \%$ in the diet group, $41.1 \%$ in the exercise group, and $46 \%$ in the combination diet and exercise group [6]. Fourteen years after the end of this trial, the lifestyle group showed a significant reduction in severe diabetic retinopathy [19], with subsequent decrease in all-cause and cardiovascular deaths 3 years later [11]. The Finnish Diabetes Prevention Study included 522 overweight prediabetic patients allocated to lifestyle intervention or usual care groups. After 3 years, the incidence of diabetes in the intervention group was 58\% lower than the control group [7]. One study examined the risk of all-cause or cardiovascular mortality associated with metabolic syndrome, obesity and CRF in a sample of 19,173 men stratified by BMI, of which 3,745 had metabolic syndrome at baseline. The presence of obesity and metabolic syndrome was associated with an increased risk of all-cause as well as cardiovascular-related mortality as compared to healthy patients. The authors did note, however, that inclusion of CRF as a covariate resulted in the disappearance of differences in relative risk of all-cause mortality and attenuation in the relative risk estimates of CVD to non-significance [20].

In another study, the role of CRF as a predictor of cancer mortality among men with prediabetes and diabetes finding that overall, higher levels of CRF were associated with a lower risk of cancer mortality as compared to those with lower levels of fitness in both prediabetic patients as well as diabetic patients. Additionally, similar trends with all-cause mortality were seen in both prediabetes patients as well as diabetes patients [21].

Mechanistically, enhanced fitness in prediabetics has been shown to have salutary impact on a multitude of cardiovascular risk parameters and provides the best opportunity for a durable therapeutic impact [22]. Our study is unique in that it associates exercise capacity with mortality risk in a veteran population, a cohort with high co-morbidity, high medication utilization and inactivity [23]. It is further strengthened by the utilization of the Veterans Health Information Database along with a patient population who receives high quality care without regard for socioeconomic status. This equal access to care irrespective of financial status [24] permits epidemiologic evaluations while minimizing the influence of medical care disparities [25]. Nevertheless, there are a number of limitations including the retrospective design, the observational aspect and the possibility that some patients were lost to follow-up. We looked at baseline BMI and CRF level at the time of the routine exercise stress test, but did not account for any weight changes or fitness level changes over time. Additionally, the actual physical activity patients engaged in and changes in glycemic control were not accounted for over time as well. Additionally, our study did not note mortality specifically due to CVD, although it was noted that the presence of CVD does not necessarily translate into mortality due to cardiovascular etiology. Future studies could be done to validate the predicted fitness mortality risk determined by this study with the 10-year Framingham Risk Score to determine the clinical utility of CRF at baseline.

\section{Conclusions}

In summary, this study demonstrates that a significant mortality benefit is associated with baseline fitness levels with the most substantial relative reduction in mortality occurring with comparison of low-fit to least-fit individuals. With increasing incidence of diabetes, prediabetic patients are considered a novel group to target with a preventative approach through exercise. This study underscores the importance of enhanced $\mathrm{CRF}$ in individuals with prediabetes.

\section{Conflict of Interest}

The authors have no conflicts of interest to disclose.

\section{References}

1. Centers for Disease Control and Prevention. National diabetes fact sheet: national estimates and general information on diabetes and prediabetes in the United States, 2011. Atlanta, GA: U.S. Department of Health and Human Services, Centers for Disease Control and Prevention, 2011.

2. Sherwin R, Jastreboff AM. Year in diabetes 2012: The diabetes tsunami. J Clin Endocrinol Metab. 2012;97(12):4293-4301.

3. Knowler WC, Barrett-Connor E, Fowler SE, Hamman RF, Lachin JM, Walker EA, Nathan DM. Reduction in the incidence of type 2 diabetes with lifestyle intervention or metformin. N Engl J Med. 2002;346(6):393-403.

4. Perreault L, Pan Q, Mather KJ, Watson KE, Hamman RF, Kahn SE. Effect of regression from prediabetes to normal glucose regulation on long-term reduction in diabetes risk: results from the Diabetes Prevention Program Outcomes Study. Lancet. 2012;379(9833):2243-2251.

5. Eriksson KF, Lindgarde F. Prevention of type 2 (non-insulin-dependent) diabetes mellitus by diet and physical exercise. The 6-year Malmo feasibility study. Diabetologia. 1991;34(12):891-898.

6. Pan XR, Li GW, Hu YH, Wang JX, Yang WY, An ZX, 
$\mathrm{Hu} \mathrm{ZX}$, et al. Effects of diet and exercise in preventing NIDDM in people with impaired glucose tolerance. The Da Qing IGT and Diabetes Study. Diabetes Care. 1997;20(4):537-544.

7. Lindstrom J, Louheranta A, Mannelin M, Rastas M, Salminen V, Eriksson J, Uusitupa M, et al. The Finnish Diabetes Prevention Study (DPS): Lifestyle intervention and 3-year results on diet and physical activity. Diabetes Care. 2003;26(12):3230-3236.

8. Norris SL, Zhang X, Avenell A, Gregg E, Schmid CH, Lau J. Long-term non-pharmacological weight loss interventions for adults with prediabetes. Cochrane Database Syst Rev. 2005;2:CD005270.

9. Norris SL, Zhang X, Avenell A, Gregg E, Bowman B, Schmid CH, Lau J. Long-term effectiveness of weightloss interventions in adults with pre-diabetes: a review. Am J Prev Med. 2005;28(1):126-139.

10. Ryden L, Standl E, Bartnik M, Van den Berghe G, Betteridge $\mathrm{J}$, de Boer MJ, Cosentino F, et al. Guidelines on diabetes, pre-diabetes, and cardiovascular diseases: executive summary. The Task Force on Diabetes and Cardiovascular Diseases of the European Society of Cardiology (ESC) and of the European Association for the Study of Diabetes (EASD). Eur Heart J. 2007;28(1):88-136.

11. Li G, Zhang P, Wang J, An Y, Gong Q, Gregg EW, Yang $\mathrm{W}$, et al. Cardiovascular mortality, all-cause mortality, and diabetes incidence after lifestyle intervention for people with impaired glucose tolerance in the Da Qing Diabetes Prevention Study: a 23-year follow-up study. Lancet Diabetes Endocrinol. 2014;2(6):474-480.

12. McAuley PA, Artero EG, Sui X, Lavie CJ, Almeida MJ, Blair SN. Fitness, fatness, and survival in adults with prediabetes. Diabetes Care. 2014;37(2):529-536.

13. Foster C, Jackson AS, Pollock ML, Taylor MM, Hare J, Sennett SM, Rod JL, et al. Generalized equations for predicting functional capacity from treadmill performance. Am Heart J. 1984;107(6):1229-1234.

14. Kokkinos P, Myers J, Nylen E, Panagiotakos DB, Manolis A, Pittaras A, Blackman MR, et al. Exercise capacity and all-cause mortality in African American and Caucasian men with type 2 diabetes. Diabetes Care. 2009;32(4):623628.

15. Myers J, Prakash M, Froelicher V, Do D, Partington S, Atwood JE. Exercise capacity and mortality among men referred for exercise testing. N Engl J Med.
2002;346(11):793-801.

16. Kokkinos P, Myers J. Exercise and physical activity: clinical outcomes and applications. Circulation. 2010;122(16):1637-1648.

17. Colberg SR, Sigal RJ, Fernhall B, Regensteiner JG, Blissmer BJ, Rubin RR, Chasan-Taber L, et al. Exercise and type 2 diabetes: the American College of Sports Medicine and the American Diabetes Association: joint position statement. Diabetes Care. 2010;33(12):e147-167.

18. Hordern MD, Dunstan DW, Prins JB, Baker MK, Singh MA, Coombes JS. Exercise prescription for patients with type 2 diabetes and pre-diabetes: a position statement from Exercise and Sport Science Australia. J Sci Med Sport. 2012;15(1):25-31.

19. Gong Q, Gregg EW, Wang J, An Y, Zhang P, Yang W, $\mathrm{Li} \mathrm{H}$, et al. Long-term effects of a randomised trial of a 6-year lifestyle intervention in impaired glucose tolerance on diabetes-related microvascular complications: the China Da Qing Diabetes Prevention Outcome Study. Diabetologia. 2011;54(2):300-307.

20. Katzmarzyk PT, Church TS, Janssen I, Ross R, Blair SN. Metabolic syndrome, obesity, and mortality: impact of cardiorespiratory fitness. Diabetes Care. 2005;28(2):391397.

21. Thompson AM, Church TS, Janssen I, Katzmarzyk PT, Earnest CP, Blair SN. Cardiorespiratory fitness as a predictor of cancer mortality among men with pre-diabetes and diabetes. Diabetes Care. 2008;31(4):764-769.

22. Sen S, Witkowski S, Lagoy A, Islam AM. A six-week exercise program improves endothelial function and CD34+ circulating progenitor cells in patients with pre-diabetes. J Endorcinol Metab 2015;5:163-171.

23. Ashton CM, Septimus J, Petersen NJ, Souchek J, Menke TJ, Collins TC, Wray NP. Healthcare use by veterans treated for diabetes mellitus in the Veterans Affairs medical care system. Am J Manag Care. 2003;9(2):145-150.

24. Selim AJ, Berlowitz DR, Fincke G, Rosen AK, Ren XS, Christiansen CL, Cong Z, et al. Risk-adjusted mortality rates as a potential outcome indicator for outpatient quality assessments. Med Care. 2002;40(3):237-245.

25. Wei M, Kampert JB, Barlow CE, Nichaman MZ, Gibbons LW, Paffenbarger RS, Jr., Blair SN. Relationship between low cardiorespiratory fitness and mortality in normal-weight, overweight, and obese men. JAMA. 1999;282(16):1547-1553. 